\title{
Problematische soziale Situation begünstigt Manifestation
}

? Im Barmer GEK Report zeigte sich, dass bei Akademikerkindern seltener eine ADHS-Diagnose gestellt wird als bei Kindern von Eltern mit niedrigeren Bildungsabschlüssen und dass besonders häufig Kinder sehr junger Eltern betroffen sind. Wie bewerten Sie diese Ergebnisse?

Dr. Maik Herberhold: Zunächst ist festzuhalten, dass meines Erachtens auf der Basis der Datenlage der Barmer GEK keine validen Aussagen zur Verteilung von ADHS in der Gesamtbevölkerung getroffen werden können. Sollte sich hier aber eine Tendenz abzeichnen, würde mich dies nicht wundern, da eine Vielzahl von psychischen Er- krankungen verstärkt bei "Unterschicht"Patienten oder -Familien auftritt. Neben den bekannten genetischen Dispositionen, die betroffenen Kindern wie auch Eltern den gesellschaftlichen Erfolg erschweren, ist denkbar, dass problematische soziale Situationen bei vorliegender zum Beispiel genetischer Vulnerabilität die Manifestation einer psychischen Störung begünstigen. Außerdem sind die Bewältigungsressourcen bei niedrigerer Bildung, prekären Lebensumständen und auch bei sehr jungen Eltern möglicherweise erheblich vermindert. Allerdings manifestiert sich die Störung vor allem im schulischen Kontext, sodass hier doch ein komplexeres Bedingungsgefüge vorliegen muss, als durch die Verkürzung

\section{ADHS-Hochburg Unterfranken?}

Stellungnahme der bayerischen Landesärzte für geistig und seelisch behinderte Kinder und Jugendliche vom 6. Feburar 2013 zum Barmer GEK Arztreport 2013

[...] Die Zahlen für die Region Unterfranken können anhand der vorliegenden Datenlage nur eingeschränkt bewertet werden. Die regionale Versorgungsstruktur muss genau analysiert werden, genauso wie die vorhandenen Daten der Barmer GEK. Die statistische Methodik wurde unzureichend angegeben, ebenso wie nur ausgewählte Zahlen der Analyse genannt werden. Eine seriöse Überprüfung der Daten ist daher schwer möglich. Zu klären sind unter anderem folgende Punkte:

— Führt die gute Versorgungsstruktur mit einem hochkompetenten universitären Zentrum und zwei Versorgungskliniken in Kombination mit einer hohen Zahl an niedergelassenen Fachärzten für Kinder- und Jugendpsychiatrie zu einer höheren Diagnoserate von ansonsten unerkannten Patienten?

_ Inwiefern ist die für Unterfranken überdurchschnittlich hohe Rate an Diagnosecodierungen bei Hausärzten erklärbar? Nicht unüblich ist, dass die Diagnose bei einem Facharzt für Kinder- und Jugendpsychiatrie gestellt wird und die Verordnungen und Kontrollen dann beim Kinderarzt oder Hausarzt erfolgen, dieser also nur die fachärztliche Diagnose übernimmt.

_ Ist bei einer vergleichsweise guten ambulanten Versorgungsstruktur wie in Unterfranken davon auszugehen, dass ein engmaschigeres Follow-up der Kinder erfolgt? Im Umkehrschluss müsste geprüft werden, ob in Regionen niedriger Versorgungsdichte diagnostizierte Patienten unzureichend begleitet und im Verlauf versorgt werden.

— Sind in der Region überproportional viele Einrichtungen der Jugendhilfe, in der auch überregional Kinder und Jugendliche mit ADHS betreut werden und regelmäßig von einem Facharzt gesehen werden? Mit Jugendhilfeeinrichtungen in Unterfranken bestehen intensive konsiliarische Liaisondienste durch Fachärzte und der Universitätsklinik, um die Gruppe von Hochrisikokindern besser zu versorgen. [...]

Prof. M. Noterdaeme, Prof. M. Romaons, Dr. M. von Aster, Prof. F. J. Freisleder, Dr. K. Holstein, Dr. M. Linder, Dr. A. Meyer

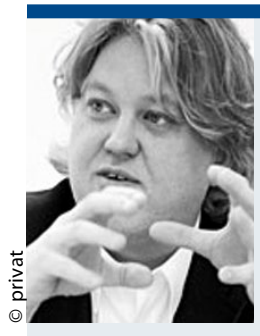

Dr. med. Maik

Herberhold

Facharzt für Kinderund Jugendpsychiatrie/Psychotherapie, Köln, Vorsitzender des Berufsverbandes für Kinder- und Jugendpsychiatrie, Psychosomatik und Psychotherapie in Deutschland e.V.

auf elterliche Erziehung etc. nahegelegt wird. Beispielsweise müsste auch untersucht werden, ob ADHS-Kinder im heutigen Bildungssystem überfordert werden; das stellt nicht den Krankheitswert der ADHS, sondern nur die jetzige Reaktion auf die Erkrankung infrage.

? Wird Ihrer Meinung nach denn zu schnell oder zu oft medikamentös behandelt?

Herberhold: Hier stehe ich als bekennender Psychotherapeut in einem Dilemma: Leider belegen alle bisherigen Untersuchungen keine ausreichende Wirksamkeit von psychotherapeutischen Verfahren oder Elterntrainings auf die Reduktion der ADHS-Kernsymptomatik. Auf jeden Fall ist aber festzustellen, dass die Bewältigung der Erkrankung sowohl für die kleinen Patienten als auch deren Eltern und Lehrer verbessert werden kann. Das heißt: Die Folgen der ADHS können gemildert werden. Viele ADHS-Patienten haben Begleiterkrankungen wie Depressionen oder Zwangsstörungen, die durch eine Psychotherapie erheblich verbessert oder geheilt werden können. Insofern ist eine multidimensionale Behandlung Pflicht, auch im Hinblick auf die Vermeidung chronifizierter Störungen im Erwachsenenalter. Hierzu gibt es gute Untersuchungen, die belegen, dass unbehandelte ADHS-Patienten beispielsweise ein achtfach erhöhtes Risiko für eine Suchterkrankung haben und überdurchschnittlich häufig als Erwachsene an schweren psychischen Störungen bis hin zu Persönlichkeitsstörungen und Psychosen leiden. 